\title{
Prepregnancy renal function and risk of preterm birth and related outcomes
}

\author{
Ziv Harel MD MSc, Alison L. Park MSc, Eric McArthur MSc, Michelle Hladunewich MD MSc, Jade S. Dirk BSc, \\ Ron Wald MDCM MPH, Amit X. Garg MD PhD, Joel G. Ray MD MSc
}

Cite as: CMAJ 2020 July 27;192:E851-7. doi: 10.1503/cmaj.200089

\begin{abstract}
BACKGROUND: Prepregnancy kidney dysfunction has been associated with preterm birth, which is the leading cause of neonatal morbidity and mortality; however, the relation is not well understood. We determined the risk of preterm birth in women with prepregnancy kidney dysfunction, defined using pregnancy-specific serum creatinine cut points.
\end{abstract}

METHODS: This population-based cohort study in the province of Ontario, Canada, involved women aged 16 to 50 years who had a singleton birth between 2006 and 2016 and measurement of serum creatinine within 10 weeks preceding their estimated conception date. The exposure was abnormally elevated prepregnancy serum creatinine, defined as greater than the 95th percentile ( $>77 \mu \mathrm{mol} / \mathrm{L})$, a value derived from a population-based sample of women without known kidney disease who became pregnant soon after the measurement was obtained. The main outcome was any preterm birth from 23 to 36 weeks' gestation. Secondary outcomes included providerinitiated preterm birth before 37 weeks' gestation and spontaneous preterm birth before 37 weeks.

RESULTS: Among 55946 pregnancies, preterm birth before 37 weeks' gestation occurred in 3956 women (7.1\%). The risk of preterm birth before 37 weeks was higher among women with prepregnancy creatinine above the 95th percentile, relative to those with prepregnancy creatinine at or below the 95th percentile $(9.1 \%$ v. $7.0 \%$; adjusted relative risk [RR] 1.23, 95\% confidence interval [CI] 1.09 to 1.38 ). The effect was significant for provider-initiated preterm birth (adjusted RR 1.30, 95\% CI 1.11 to 1.52 ) but not for spontaneous preterm birth (adjusted RR 1.12, 95\% Cl 0.91 to 1.37 ).

INTERPRETATION: Given that prepregnancy kidney dysfunction conferred an increased risk of preterm birth, measurement of serum creatinine (a relatively inexpensive blood test) may form part of the assessment of risk for preterm birth among those planning pregnancy.

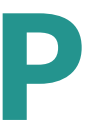

repregnancy kidney dysfunction may perturb the normal physiologic adaptations of pregnancy, predisposing a woman and her fetus to adversity, at least partly mediated by placental and endothelial dysfunction. ${ }^{1}$ Complications such as preeclampsia ${ }^{2}$ and poor fetal growth ${ }^{3}$ may necessitate provider-initiated preterm birth. Preterm birth of any form before 37 weeks' gestation occurs in $6 \%$ to $11 \%$ of viable pregnancies and is the leading cause of infant death. ${ }^{4}$

Prepregnancy kidney dysfunction has been associated with preterm birth. ${ }^{5-7}$ Prior studies of the relation between prepregnancy kidney dysfunction and preterm birth were primarily case series and thus had inadequate statistical power to differentiate between the outcomes of spontaneous versus providerinitiated preterm birth. In addition, arbitrary cut points were used in these studies to define prepregnancy kidney dysfunction, and there was no accounting for important confounders. ${ }^{5,8-14}$
In an effort to overcome the aforementioned limitations, we completed a large cohort study in a setting where prenatal and obstetric care is covered under a provincial health insurance plan. Using population-derived cut points for prepregnancy serum creatinine to define kidney dysfunction, we examined the risk of preterm birth and other related outcomes.

\section{Methods}

\section{Study design and data sources}

In this population-based cohort study, we considered all live births and stillbirths that occurred in hospital in Ontario, Canada, from April 2007 to October 2016. Obstetric care is covered for Ontario residents through the Ontario Health Insurance Plan, and nearly all women undergo ultrasonography during the first or second trimester to enable accurate dating of the pregnancy. ${ }^{15}$ 
We identified all hospital births in Ontario in the Canadian Institute for Health Information's Discharge Abstract Database, and linked these records to the Ontario Laboratories Information System (https://ehealthontario.on.ca/images/uploads/pages/ documents/Lab_Results.pdf), which captures most outpatient laboratory testing in Ontario. We standardized all serum creatinine values obtained from the Ontario Laboratories Information System to isotope dilution mass spectrometry, the current reference standard. ${ }^{16}$ We linked all data sets using unique encoded identifiers for analysis at ICES. Specifics about the study databases are shown in Appendix 1, Table A1 (available at www.cmaj. ca/lookup/suppl/doi:10.1503/cmaj.200089/-/DC1).

\section{Participants}

Eligible participants were women with an in-hospital obstetric delivery between April 2007 and October 2016 in Ontario, with at least 1 outpatient measurement of serum creatinine within 10 weeks before the estimated date of conception. We chose a 10-week cut-off for serum creatinine measurement because there exist validated population-based values for this metric during that time period among healthy women who soon became pregnant. ${ }^{17}$ We excluded those younger than 16 years or older than 50 years at the time of conception, those with multifetal pregnancies and those with end-stage renal disease or a kidney transplant before conception. We also excluded women who died during pregnancy and those with a miscarriage, ectopic pregnancy or induced abortion before 20 weeks' gestation.

\section{Exposures and outcomes}

The main exposure of interest was outpatient measurement of serum creatinine within 10 weeks before the estimated date of conception. For women with multiple serum creatinine measurements, we used the one closest to the time of conception. We defined abnormally elevated prepregnancy serum creatinine as any value above the 95th percentile (i.e., > $77 \mu \mathrm{mol} / \mathrm{L}$ ), previously derived from a population-based sample of women without known kidney disease who became pregnant soon after the measurement. ${ }^{17}$

The primary outcome was any preterm birth from 23 to 36 weeks' gestation. Secondary outcomes were provider-initiated preterm birth at less than 37 weeks' gestation, spontaneous preterm birth at less than 37 weeks' gestation, early preterm birth (<32 weeks' gestation), preterm birth at less than 37 weeks' gestation with newborn severely small for gestational age (i.e., below the 5th percentile for sex and gestational age), preterm birth at less than 37 weeks' gestation with concomitant preeclampsia, and stillbirth at or later than 20 weeks' gestation. The diagnostic codes used to identify each study outcome, along with information on their validation (including identification of a subcohort of women with chronic kidney disease), are detailed in Appendix 1, Table A2..

\section{Statistical analysis}

We first explored the continuous association between serum creatinine and the relative risk of preterm birth at less than 37 weeks' gestation using univariable fractional polynomials in a modified Poisson regression model. We selected fractional polynomials using the "mfp" package (https://CRAN.R-project.org/package=mfp) in R soft- ware, version 3.1.2 (R Foundation, 2014), which uses the RA2 selection algorithm to select the best fitting of 44 models with different combinations of power transformations of the explanatory variable. ${ }^{18,19}$ On average, fractional polynomials show less bias and more precision than restricted cubic splines. Modified Poisson regression directly estimates the relative risk (RR) of the binary outcome and is a popular alternative to logistic regression models. ${ }^{20}$ After inspection of the best-fit plot, we subsequently categorized serum creatinine as either above the 95th percentile or less than or equal to the 95th percentile to facilitate interpretation of the model results.

Using the main model, we examined the associated risk of preterm birth and the secondary outcomes, each in relation to a prepregnancy serum creatinine value above the 95th percentile. We used modified Poisson regression with a robust error variance to derive unadjusted and adjusted RR values and 95\% confidence intervals (Cls) for each study outcome. For women who had more than 1 pregnancy, we used generalized estimating equations with an exchangeable correlation structure to account for correlated errors between pregnancies. We adjusted the RRs for variables chosen a priori, based on the existing literature, and included maternal age (continuous), rural residence (rural/missing or urban), residential income quintile (Q1/missing, Q2, Q3, Q4 or Q5 [highest]), world region of origin (Asia, Caribbean/Africa, Hispanic America or other) - each at the time of the index conception - as well as diabetes mellitus, chronic hypertension, and use of tobacco or illicit drugs within 4 years before the index conception date.

As might be expected for women of child-bearing age, estimated glomerular filtration rate is almost entirely dictated by the serum creatinine value. Accordingly, for all analyses, we used serum creatinine as the measure of kidney function. ${ }^{21}$

We performed several additional analyses. To assess the difference in outcomes between women with and without prepregnancy impairment of kidney function (i.e., chronic kidney disease), we stratified the main model by these 2 subcohorts. Among women with chronic kidney disease, we further adjusted the multivariable model examining the outcome of preterm birth with concomitant preeclampsia for prepregnancy proteinuria, because new-onset proteinuria in pregnancy is itself a diagnostic criterion for preeclampsia (additional analysis 1).

Serum creatinine is not routinely assessed before or during pregnancy. As such, a woman could undergo serum creatinine measurement for a pre-existing condition, which might impart a selection bias. To assess for such a bias, we randomly selected women without prepregnancy measurement of serum creatinine from all who gave birth and matched them 1:1 by year of pregnancy to the main cohort of women with prepregnancy measurement of serum creatinine. We then reran the main model to compare those with and those without measurement of prepregnancy serum creatinine, while accounting for matching (additional analysis 2).

A pregnancy may not be planned, so a woman may have serum creatinine measured only in early pregnancy, such as at the time of her routine prenatal biochemical serum screening, typically at $11^{0 / 7}$ to $20^{6 / 7}$ weeks' gestation. Accordingly, we assessed the relation between serum creatinine measured before pregnancy and at $11^{0 / 7}$ up to $20^{6 / 7}$ weeks' gestation using a Pearson correlation coefficient, limiting this analysis to the 5262 women whose serum creatinine was 
measured at both time periods. Because the Pearson correlation was high $(0.84,95 \% \mathrm{Cl} 0.84$ to 0.85$)$, we reran the main model for all study outcomes in a cohort of women who had serum creatinine measured between $11^{0 / 7}$ and $20^{6 / 7}$ weeks' gestation, using a lower serum creatinine 95th percentile cut point ( $>59 \mu \mathrm{mol} / \mathrm{L})$. This lower cut point, which reflects the natural decline in serum creatinine during those gestational weeks, was also derived in a large population-based sample of women without apparent kidney dysfunction ${ }^{17}$ (additional analysis 3).

To account for potential surveillance bias imparted by a prior pregnancy, we reran the main model among nulliparous women (additional analysis 4). Additional analysis 5 was restricted to those with a recorded prepregnancy weight, which was further added to the main model as a covariable.

Finally, we repeated the main analysis with abnormal prepregnancy serum creatinine defined as greater than the 99th percentile (i.e., $>86 \mu \mathrm{mol} / \mathrm{L}$ ), reflective of potentially worse renal function ${ }^{17}$ (additional analysis 6).

We used SAS version 9.4 for UNIX for all statistical analyses.

\section{Ethics approval}

The use of data in this project was authorized under section 45 of Ontario's Personal Health Information Protection Act, which does not require review by a research ethics board.

\section{Results}

There were 57359 pregnancies in which serum creatinine was measured in the 10-week period before conception. Of these, 1413 (2.5\%) were excluded, leaving 55946 pregnancies in the main cohort (Figure 1 and Table 1 ). The mean maternal age was 30.7 years, and $45.7 \%$ were nulliparous.

In the subcohort of 9830 pregnancies in women with chronic kidney disease, 8945 (91.0\%) had a urine dipstick test for proteinuria, with the following results: no proteinuria (5253 [58.7\%]); trace proteinuria (2071 [23.2\%]); 1 to $2+$ proteinuria (1416 [15.8\%]); 3+ proteinuria (163 [1.8\%]); missing (42 (0.47\%]). Of these 9830 pregnancies in women with chronic kidney disease, $3303(33.6 \%)$ had a urine microalbumin to creatinine ratio test, with the following results: undetectable to $\leq 2.0$ (1487 [45.0\%]); 2.1 to 20 (1547 [46.8\%]); > 20 (256 [7.8\%]); missing (13 [0.39\%]).

For the entire cohort, there was a J-shaped relation between serum creatinine and the probability of preterm birth before 37 weeks' gestation (Appendix 2, available at www.cmaj.ca/ lookup/suppl/doi:10.1503/cmaj.200089/-/DC1). The lower 95\% Cl for the risk of preterm birth became apparent starting at a serum creatinine of about $75 \mu \mathrm{mol} / \mathrm{L}$.

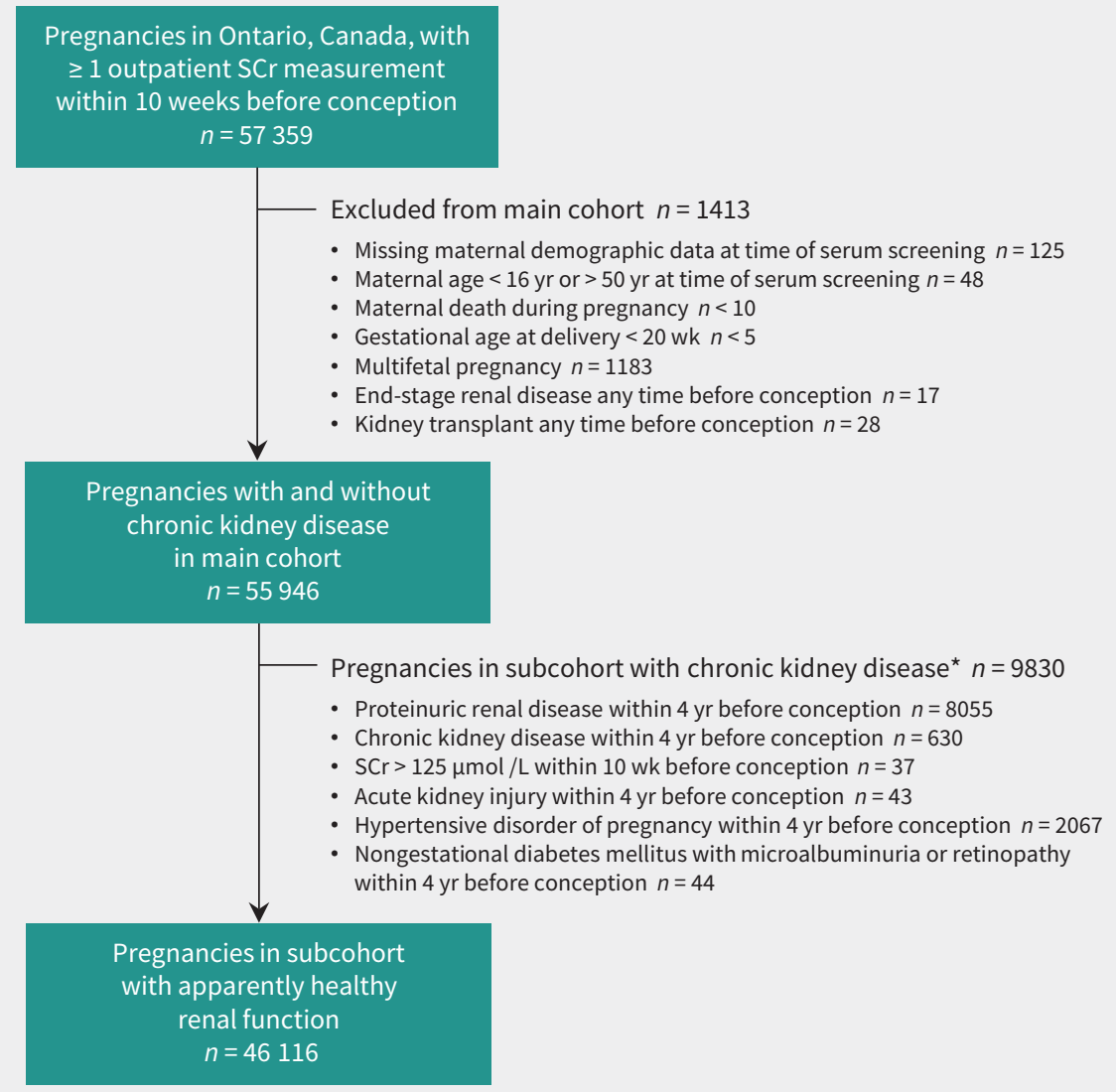

Figure 1: Study flow diagram for creation of the main cohort of pregnancies with measurement of serum creatinine ( $\mathrm{SCr}$ ) within 10 weeks before conception, as well as the subcohort of pregnancies with apparently healthy renal function at the time of conception. *In the subcohort with chronic kidney disease, the frequencies of individual conditions are nonexclusive. 
The primary outcome of preterm birth before 37 weeks' gestation was more frequent among women with prepregnancy serum creatinine above the 95th percentile (254 events [9.1\%]) than among those whose serum creatinine was normal (3702 events [7.0\%]), for a crude RR of $1.28(95 \% \mathrm{Cl} 1.14$ to 1.45$)$ and an adjusted RR of 1.23 (95\% Cl 1.09 to 1.38) (Table 2). Of all preterm births, 2255 (57.0\%) were initiated by the provider and 1701 (43.0\%) were spontaneous. The adjusted RR for preterm birth before 37 weeks' gestation was significant for provider-initiated preterm birth $(1.30,95 \% \mathrm{Cl} 1.11$ to 1.52$)$ but not for spontaneous preterm birth (1.12, 95\% $\mathrm{Cl} 0.91$ to 1.37$)$ (Table 2). The outcome of early preterm birth (before 32 weeks' gestation) occurred among $2.0 \%$ of women with elevated prepregnancy serum creatinine compared with $0.93 \%$ of those at or below the 95th percentile (adjusted RR 2.04, 95\% Cl 1.54 to 2.69). All other outcomes followed a similar pattern (Table 2).

Of the 55946 pregnancies in the main cohort, 46116 occurred in women who had apparently healthy prepregnancy kidney function and 9830 in women who had chronic kidney disease (Figure 1 and Table 1). Women in the subcohort with chronic kidney disease

Table 1: Characteristics of pregnancies with measurement of serum creatinine within 10 weeks before conception

\begin{tabular}{|c|c|c|c|}
\hline \multirow[b]{2}{*}{ Characteristic } & \multicolumn{3}{|c|}{ Cohort or subcohort; no. (\%) of pregnancies* } \\
\hline & $\begin{array}{c}\text { Overall } \\
n=55946\end{array}$ & $\begin{array}{l}\text { With apparently healthy } \\
\text { renal function } \\
n=46116\end{array}$ & $\begin{array}{l}\text { With chronic kidney } \\
\text { disease } † \\
n=9830\end{array}$ \\
\hline Age at time of conception, $y r$, mean \pm SD & $30.7 \pm 5.1$ & $30.7 \pm 5.1$ & $30.5 \pm 5.4$ \\
\hline \multicolumn{4}{|l|}{ Maternal world region of origin } \\
\hline Asia & $10982(19.6)$ & $9252(20.1)$ & $1730(17.6)$ \\
\hline Caribbean/Africa & $2542(4.5)$ & $1936(4.2)$ & $606(6.2)$ \\
\hline Hispanic America & $1520(2.7)$ & $1256(2.7)$ & $264(2.7)$ \\
\hline Other & $40902(73.1)$ & $33672(73.0)$ & $7230(73.6)$ \\
\hline \multicolumn{4}{|l|}{ Income quintile } \\
\hline Q1 (lowest) or missing & $12014(21.5)$ & $9628(20.9)$ & $2386(24.3)$ \\
\hline Q2 & $11225(20.1)$ & $9240(20.0)$ & $1985(20.2)$ \\
\hline Q3 & $11306(20.2)$ & $9311(20.2)$ & $1995(20.3)$ \\
\hline Q4 & $12455(22.3)$ & $10358(22.5)$ & $2097(21.3)$ \\
\hline Q5 (highest) & $8946(16.0)$ & $7579(16.4)$ & $1367(13.9)$ \\
\hline Urban residence & $52563(94.0)$ & $43292(93.9)$ & $9271(94.3)$ \\
\hline Parity, median (IQR) & $1(0-1)$ & $1(0-1)$ & $1(0-1)$ \\
\hline Nulliparous & $25563(45.7)$ & $22205(48.2)$ & $3358(34.2)$ \\
\hline Prepregnancy weight, kg,mean \pm SD $\ddagger$ & $69.8 \pm 17.9$ & $69.2 \pm 17.4$ & $72.7 \pm 19.7$ \\
\hline \multicolumn{4}{|l|}{ Medical comorbidities $\leq 4 \mathrm{yr}$ before conception } \\
\hline Diabetes mellitus type 1 and type 2 & $8695(15.5)$ & $6456(14.0)$ & $2239(22.8)$ \\
\hline Chronic hypertension & 7785 (13.9) & $5324(11.5)$ & $2461(25.0)$ \\
\hline Illicit drug use or tobacco use & $3016(5.4)$ & $2442(5.3)$ & $574(5.8)$ \\
\hline \multicolumn{4}{|l|}{ Maternal conditions during index pregnancy } \\
\hline Hypertensive disorder of pregnancy & $3387(6.1)$ & $2359(5.1)$ & $1028(10.5)$ \\
\hline Gestational diabetes mellitus & $5225(9.3)$ & $3713(8.1)$ & $1512(15.4)$ \\
\hline \multicolumn{4}{|l|}{ Measured renal function before conception } \\
\hline \multicolumn{4}{|l|}{ Serum creatinine } \\
\hline Measured value, $\mu \mathrm{mol} / \mathrm{L}$, mean $\pm \mathrm{SD}$ & $60.7 \pm 10.4$ & $60.4 \pm 9.5$ & $61.7 \pm 14.1$ \\
\hline Measured value > 95th percentile (i.e., > 77 $\mu \mathrm{mol} / \mathrm{L}$ ) & $2831(5.1)$ & $2105(4.6)$ & $726(7.0)$ \\
\hline Measured value > 99th percentile (i.e., > 86 mol/L) & $684(1.2)$ & $413(0.90)$ & $271(2.8)$ \\
\hline \multicolumn{4}{|l|}{ Baseline eGFR } \\
\hline Measured value, $\mathrm{mL} / \mathrm{min} / 1.73 \mathrm{~m}^{2}$, mean $\pm \mathrm{SD}$ & $113.9 \pm 13.8$ & $114.0 \pm 13.3$ & $113.1 \pm 15.8$ \\
\hline Measured value $>60 \mathrm{~mL} / \mathrm{min} / 1.73 \mathrm{~m}^{2}$ & $55858(99.9)$ & $46116(100.0)$ & $9742(99.1)$ \\
\hline Measured value $<5$ th percentile (i.e., $<88 \mathrm{~mL} / \mathrm{min} / 1.73 \mathrm{~m}^{2}$ ) & $2824(5.0)$ & $2135(4.6)$ & $689(7.0)$ \\
\hline
\end{tabular}


had a higher burden of comorbid conditions than those with apparently healthy renal function (Table 1 ).

In an examination of the 2 subcohorts, there was a higher rate of preterm birth before 37 weeks' gestation among those with prepregnancy chronic kidney disease than among those with apparently healthy renal function, including those without elevated serum creatinine $(9.7 \%$ and $6.5 \%$, respectively) (additional analysis 1, presented in Appendix 3, available at www.cmaj.ca/lookup/suppl/ doi:10.1503/cmaj.200089/-/DC1). Stratification by these subcohorts generally showed more prominent and significant adjusted RRs related to abnormally high serum creatinine among the women with prepregnancy chronic kidney disease than among those without this condition, especially for the outcomes of preterm birth with concomitant preeclampsia (adjusted RR 2.52, 95\% Cl 1.68 to 3.77 ) and stillbirth (adjusted RR 3.14, 95\% Cl 1.76 to 5.61) (Appendix 3).

In the matched analysis of women who did and did not have measured prepregnancy serum creatinine, the adjusted RR for preterm birth before 37 weeks' gestation was 1.05 (95\% Cl 1.01 to 1.10) (additional analysis 2, presented in Appendix 4, available at www.cmaj.ca/lookup/suppl/doi:10.1503/cmaj.200089/-/ DC1). Other than provider-initiated preterm birth, no differences were seen between groups for the other secondary outcomes (Appendix 4).

In a separate cohort of 56819 pregnancies in which serum creatinine was measured at $11^{0 / 7}$ to $20^{6 / 7}$ weeks' gestation, and using a 95th percentile cut point of $59 \mu \mathrm{mol} / \mathrm{L}$, the adjusted RRs for the primary outcome and most secondary outcomes were greater in magnitude than in the main analysis (additional analysis 3, presented in Appendix 5, available at www.cmaj.ca/lookup/ suppl/doi:10.1503/cmaj.200089/-/DC1).

The results of additional analyses 4, 5 and 6 are presented in Appendix 6, Appendix 7 and Appendix 8, respectively (all available at www.cmaj.ca/lookup/suppl/doi:10.1503/cmaj.200089/-/ DC1).

\section{Interpretation}

In this population-based study of nearly 56000 pregnancies, the risk of preterm birth before 37 weeks' gestation was 1.23 times higher for women with prepregnancy kidney dysfunction than for those with normal renal function. The associated risk of severe preterm birth (before 32 weeks' gestation) was about

Table 2: Risk of primary outcome (preterm birth < 37 weeks' gestation) and secondary outcomes in association with abnormal prepregnancy serum creatinine $(\mathrm{SCr})^{*}$

\begin{tabular}{|c|c|c|c|c|}
\hline Outcome† & $\begin{array}{l}\text { No. (\%) with } \\
\text { outcome }\end{array}$ & $\begin{array}{c}\text { Absolute risk difference, } \\
\%(95 \% \mathrm{Cl})\end{array}$ & \multicolumn{2}{|c|}{$\operatorname{RR}(95 \% \mathrm{Cl})$} \\
\hline SCr normal: $\leq 95$ th percentile $(n=52837)$ & $3702(7.0)$ & 0.0 (ref.) & 1.00 (ref.) & 1.00 (ref.) \\
\hline SCr abnormal: > 95th percentile $(n=2806)$ & $254(9.1)$ & 2.1 (0.96 to 3.10$)$ & $1.28(1.14$ to 1.45$)$ & 1.23 (1.09 to 1.38$)$ \\
\hline SCr abnormal: > 95th percentile $(n=2806)$ & $158(5.6)$ & $1.7(0.79$ to 2.50$)$ & $1.42(1.21$ to 1.66$)$ & 1.30 (1.11 to 1.52$)$ \\
\hline \multicolumn{5}{|l|}{ Spontaneous preterm birth $<37$ wk gestation } \\
\hline SCr normal: $\leq 95$ th percentile $(n=52837)$ & $1605(3.0)$ & 0.0 (ref.) & 1.00 (ref.) & 1.00 (ref.) \\
\hline SCr abnormal: > 95th percentile $(n=2806)$ & $96(3.4)$ & $0.38(-0.30$ to 1.10$)$ & 1.11 (0.90 to 1.36$)$ & 1.12 (0.91 to 1.37 ) \\
\hline \multicolumn{5}{|l|}{ Preterm birth $<37$ wk gestation with severe SGA } \\
\hline SCr normal: $\leq 95$ th percentile $(n=52781)$ & $200(0.38)$ & 0.0 (ref.) & 1.00 (ref.) & 1.00 (ref.) \\
\hline SCr abnormal: > 95th percentile $(n=2804)$ & $18(0.64)$ & $0.26(-0.04$ to 0.56$)$ & 1.69 (1.05 to 2.74$)$ & 1.52 (0.94 to 2.45$)$ \\
\hline \multicolumn{5}{|c|}{ Preterm birth $<37$ wk gestation with preeclampsia } \\
\hline SCr normal: $\leq 95$ th percentile $(n=52837)$ & $370(0.70)$ & 0.0 (ref.) & 1.00 (ref.) & 1.00 (ref.) \\
\hline SCr abnormal: $>$ 95th percentile $(n=2806)$ & $42(1.5)$ & $0.80(0.34$ to 1.30$)$ & 2.14 (1.56 to 2.93$)$ & 1.82 (1.33 to 2.49$)$ \\
\hline \multicolumn{5}{|l|}{ Stillbirth at $\geq 20$ wk gestation } \\
\hline SCr normal: $\leq 95$ th percentile $(n=53115)$ & $278(0.52)$ & 0.0 (ref.) & 1.00 (ref.) & 1.00 (ref.) \\
\hline SCr abnormal: $>$ 95th percentile $(n=2831)$ & $25(0.88)$ & 0.36 (0.01 to 0.71$)$ & 1.69 (1.12 to 2.55$)$ & 1.57 (1.04 to 2.37 ) \\
\hline
\end{tabular}


doubled, and other outcomes related to preterm birth and poor placentation were also seen, especially for women with chronic kidney disease.

Prior studies of women with prepregnancy kidney dysfunction have been few in number and inconsistent in their conclusions. A population-based study of 3405 Norwegian women with mean serum creatinine before pregnancy of $60.5 \mu \mathrm{mol} / \mathrm{L}$, a value identical with that reported here, showed that after a mean of 4.7 years since their baseline serum creatinine measurement, the risk of preterm birth was $6 \%$, with abnormal estimated glomerular filtration rate defined by a cut point of less than $90 \mathrm{~mL} / \mathrm{min}^{14}$ In the Norwegian study, a significantly higher risk of the combined end point of preeclampsia, small for gestational age and preterm birth was not observed for women below the cut point for estimated glomerular filtration rate (relative to those above the cut point), but the large gap in time between serum creatinine measurement and any subsequent pregnancy might have diluted any true relation or might have prevented the accurate assessment of important confounders. In contrast, in another study involving 504 pregnant Italian women, most affected by stage 1 chronic kidney disease, the observed rate of preterm birth was $33.4 \% .^{12}$ Furthermore, the observed risk of the combined outcome of preterm birth, small for gestational age below the 10th percentile and admission to neonatal intensive care was higher among those with stage 1 chronic kidney disease than among those with "normal" prepregnancy renal function (odds ratio $2.67,95 \% \mathrm{Cl} 2.00$ to 3.55 ).

The current findings underscore the importance of prepregnancy kidney dysfunction for key perinatal outcomes. It has been postulated that even mild prepregnancy kidney dysfunction can cause a maladaptive response to the normal physiologic changes expected in pregnancy, ${ }^{22}$ leading to placental dysfunction. ${ }^{23-26}$ In turn, placental dysfunction can produce a fetus that is severely small for gestational age and preeclampsia, each of which is a leading cause of provider-initiated preterm birth;,26-28 it can also cause higher neonatal morbidity and mortality. ${ }^{4,26-30}$ In the current study, elevated serum creatinine was notably associated with an increased risk for outcomes of a fetus severely small for gestational age and preeclampsia, which may be reasons for the increase in provider-initiated preterm birth but not spontaneous preterm birth.

The timely recognition of prepregnancy kidney dysfunction has several potential benefits, including informed counselling about the risk of adverse perinatal outcomes, closer monitoring of mother and fetus in pregnancy and potential use of acetylsalicylic acid to prevent preeclampsia. ${ }^{29,31}$ However, the American College of Obstetricians and Gynecologists does not specifically address prepregnancy counselling or recommend investigations for women with pre-existing renal dysfunction who are planning a pregnancy. ${ }^{32}$ It may be appropriate in future guidelines to consider the importance of serum creatinine measurement before pregnancy or during the time of prenatal serum screening, especially given the test's low cost and widespread availability. Development of patient-specific clinical prediction models for preterm birth or preeclampsia ${ }^{32}$ should likely include a test for the added value of the serum creatinine concentration.

\section{Limitations}

This study had several imitations. We could not assess for medications that might influence renal function, including nonsteroidal antiinflammatory drugs or angiotensin-converting enzyme inhibitors, nor could we assess for acute conditions, such as nephrolithiasis.

Some of the diagnostic codes for our outcomes have not been validated, specifically those for provider-initiated preterm birth and spontaneous preterm birth.

Prepregnancy renal function was based on a single measurement and thus may have been misclassified. However, serum creatinine shows little short-term within-person variability in the general population. ${ }^{33}$

The criteria used to distinguish women with apparently healthy prepregnancy renal function from those with prepregnancy chronic kidney disease may have erroneously misclassified women. In particular, there may have been assignment of women with chronic kidney disease to the group with healthy renal function, because the diagnostic codes for chronic kidney disease have low sensitivity.

The main cohort consisted of women with serum creatinine measured on an outpatient basis and thus did not include women who did not use medical services; those with serum creatinine measured before the 10-week preconception period were also omitted. To assess for this potential selection bias, we matched women with and without a prepregnancy serum creatinine and observed some minor differences in the risk of preterm birth and related outcomes (as shown in Appendix 4).

Out-of-hospital births were not captured in this study; however, they account for less than $2 \%$ of all Canadian births. ${ }^{34}$

A large proportion of women in the current cohort did not have preconception measurement of urine protein, so the relation between renal clearance and preterm birth may have been influenced by unrecognized proteinuria in some women.

The subgroup of women with chronic kidney disease was likely heterogeneous, in terms of the causes and extent of their renal disease. This heterogeneity may impart different risks to pregnancy. ${ }^{35}$

\section{Conclusion}

In this study, prepregnancy kidney dysfunction, defined according to population-based cut points, was associated with an increased risk of preterm birth, as well as other related outcomes. This was especially so for women with underlying chronic kidney disease. Given that measurement of serum creatinine is a readily available and inexpensive blood test, there may be a role for screening potentially high-risk women for kidney dysfunction either before conception or in the first half of pregnancy.

\section{References}

1. Lopes van Balen VA, van Gansewinkel TAG, de Haas S, et al. Kidney function during pregnancy: a systematic review and meta-analysis. Ultrasound Obstet Gynecol 2019;54:297-307.

2. Levine RJ, Maynard SE, Qian C, et al. Circulating angiogenic factors and the risk of preeclampsia. N Engl J Med 2004;350:672-83.

3. Mook-Kanamori DO, Steegers EA, Eilers PH, et al. Risk factors and outcomes associated with first-trimester fetal growth restriction. JAMA 2010;303:527-34.

4. Lawn JE, Kinney MV, Belizan JM, et al. Born too soon: accelerating actions for prevention and care of 15 million newborns born too soon. Reprod Health 2013;10(Suppl 1):S6 
5. Jones DC, Hayslett JP. Outcome of pregnancy in women with moderate or severe renal insufficiency. N Engl J Med 1996;335:226-32.

6. Imbasciati E, Gregorini G, Cabiddu G, et al. Pregnancy in CKD stages 3 to 5: fetal and maternal outcomes. Am J Kidney Dis 2007;49:753-62.

7. He Y, Liu J, Cai Q, et al. The pregnancy outcomes in patients with stage 3-4 chronic kidney disease and the effects of pregnancy in the long-term kidney function. J Nephrol 2018;31:953-60.

8. Katz Al, Davison JM, Hayslett JP, et al. Pregnancy in women with kidney disease. Kidney Int 1980;18:192-206.

9. Piccoli GB, Attini R, Vasario E, et al. Pregnancy and chronic kidney disease: a challenge in all CKD stages. Clin J Am Soc Nephrol 2010;5:844-55.

10. Jungers $P$, Chauveau $D$, Choukroun $G$, et al. Pregnancy in women with impaired renal function. Clin Nephrol 1997;47:281-8.

11. Cunningham FG, Cox SM, Harstad TW, et al. Chronic renal disease and pregnancy outcome. Am J Obstet Gynecol 1990;163:453-9.

12. Piccoli GB, Cabiddu G, Attini R, et al. Risk of adverse pregnancy outcomes in women with CKD. J Am Soc Nephrol 2015;26:2011-22.

13. Davidson NL, Wolski $P$, Callaway LK, et al. Chronic kidney disease in pregnancy: maternal and fetal outcomes and progression of kidney disease. Obstet Med 2015;8:92-8.

14. Munkhaugen J, Lydersen S, Romundstad PR, et al. Kidney function and future risk for adverse pregnancy outcomes: a population-based study from HUNT II, Norway. Nephrol Dial Transplant 2009;24:3744-50.

15. You JJ, Alter DA, Stukel TA, et al. Proliferation of prenatal ultrasonography. CMAJ 2010;182:143-51.

16. Levey AS, Coresh J, Greene T, et al. Using standardized serum creatinine values in the modification of diet in renal disease study equation for estimating glomerular filtration rate. Ann Intern Med 2006;145:247-54.

17. Harel Z, McArthur E, Hladunewich M, et al. Serum creatinine levels before, during, and after pregnancy. JAMA 2019;321:205-7.

18. Royston P, Altman D. Regression using fractional polynomials of continuous covariates: parsimonious parametric modelling (with discussion). Appl Stat 1994;43:429-67.

19. Ambler G, Royston P. Fractional polynomial model selection procedures: investigation of type I error rate. J Stat Comput Simul 2001;69:89-108.

20. Zou G. A modified Poisson regression approach to prospective studies with binary data. Am J Epidemiol 2004;159:702-6.
21. Levey AS, Stevens LA, Schmid CH, et al. A new equation to estimate glomerular filtration rate. Ann Intern Med 2009;150:604-12.

22. Odutayo A, Hladunewich M. Obstetric nephrology: renal hemodynamic and metabolic physiology in normal pregnancy. Clin J Am Soc Nephrol 2012;7:2073-80.

23. Maynard SE, Min JY, Merchan J, et al. Excess placental soluble fms-like tyrosine kinase 1 (sFlt1) may contribute to endothelial dysfunction, hypertension, and proteinuria in preeclampsia. J Clin Invest 2003;111:649-58.

24. Di Marco GS, Reuter S, Hillebrand U, et al. The soluble VEGF receptor sFlt1 contributes to endothelial dysfunction in CKD. J Am Soc Nephrol 2009;20:2235-45.

25. Stam F, van Guldener C, Becker A, et al. Endothelial dysfunction contributes to renal function-associated cardiovascular mortality in a population with mild renal insufficiency: the Hoorn study. J Am Soc Nephrol 2006;17:537-45.

26. Ananth CV, Vintzileos AM. Ischemic placental disease: epidemiology and risk factors. Eur J Obstet Gynecol Reprod Biol 2011;159:77-82.

27. Ray JG, Park AL, Fell DB. Mortality in infants affected by preterm birth and severe small-for-gestational age birth weight. Pediatrics 2017;140.pii:e20171881.

28. Garite TJ, Clark R, Thorp JA. Intrauterine growth restriction increases morbidity and mortality among premature neonates. Am J Obstet Gynecol 2004;191:481-7.

29. Ray JG, Bartsch E, Park AL, et al. Estimated reductions in provider-initiated preterm births and hospital length of stay under a universal acetylsalicylic acid prophylaxis strategy: a retrospective cohort study. CMAJ Open 2017;5:E508-16.

30. Catov JM, Scifres CM, Caritis SN, et al. Neonatal outcomes following preterm birth classified according to placental features. Am J Obstet Gynecol 2017;216: 411.e1- 411.e14.

31. van Vliet EO, Askie LA, Mol BW, et al. Antiplatelet agents and the prevention of spontaneous preterm birth: a systematic review and meta-analysis. Obstet Gynecol 2017;129:327-36.

32. ACOG Committee Opinion No. 762: prepregnancy counseling. Obstet Gynecol 2019;133:e78-89.

33. Selvin E, Juraschek SP, Eckfeldt J, et al. Within-person variability in kidney measures. Am J Kidney Dis 2013;61:716-22.

34. What mothers say: the Canadian Maternity Experiences Survey, 2009. Ottawa: Public Health Agency of Canada; 2009.

35. Cabiddu G, Castellino S, Gernone G, et al. A best practice position statement on pregnancy in chronic kidney disease: the Italian Study Group on Kidney and Pregnancy. J Nephrol 2016;29:277-303.
Competing interests: Michelle Hladunewich has received grants from Ionis Pharmaceuticals, Calliditas Therapeutics, Pfizer and ChemoCentryx for research projects outside the study reported here. In addition, she has received consulting fees from Alnylam Pharmaceuticals; serves as an editor with UpToDate, for the sections on chronic kidney disease/end-stage renal disease and pregnancy; and is the medical head for glomerulonephritis and pregnancy for the Ontario Renal Network. No other competing interests were declared.

This article has been peer reviewed.

Affiliations: Division of Nephrology (Harel, Wald) and Department of Medicine (Ray), St. Michael's Hospital, Toronto, Ont.; ICES (Park, McArthur, Dirk, Garg, Ray), Ontario; Division of Nephrology (Hladunewich), Sunnybrook Health Sciences Centre, Toronto, Ont.; Division of Nephrology (Garg), Western University, London, Ont.

Contributors: Ziv Harel, Alison Park and Joel Ray contributed to study concept and design. All of the authors acquired, analyzed or interpreted the data. Alison Park and Joel Ray performed the statistical analysis. Ziv Harel, Alison Park and Joel Ray drafted the manuscript, and all of the authors critically revised the manuscript for important intellectual content. Ziv Harel and Joel Ray obtained funding. Joel Ray also provided administrative, technical or material support and supervised the study. Alison Park had full access to all the data in the study and takes responsibility for the integrity of the data and the accuracy of the data analysis. All of the authors approved the final version for publication and agreed to be accountable for all aspects of the work.

Funding: This work was supported by a Biomedical Research Grant from the Kidney Foundation of Canada. This study was also supported by ICES, which is funded by an annual grant from the Ontario Ministry of Health and Long-Term Care (MOHLTC). Amit Garg is supported by the Dr. Adam Linton Chair in Kidney Health Analytics and a Clinician Investigator Grant from the Canadian Institutes of Health Research.

Data sharing: The data set from this study is held securely in coded form at ICES. Although data-sharing agreements prohibit ICES from making the data set publicly available, access may be granted to those who meet prespecified criteria for confidential access, available at www.ices.on.ca/DAS. The full data set, creation plan and underlying analytic code are available from the authors upon request, with the understanding that the computer programs may rely upon coding templates or macros that are unique to ICES and are therefore either inaccessible or may require modification.

Disclaimer: This study was supported by ICES, which is funded by an annual grant from the Ontario Ministry of Health and Long-Term Care (MOHLTC). The opinions, results and conclusions reported in this paper are those of the authors and are independent from the funding sources. No endorsement by ICES or the Ontario MOHLTC is intended or should be inferred. Parts of this material are based on data and/or information compiled and provided by the Canadian Institute for Health Information (CIHI). However, the analyses, conclusions, opinions and statements expressed in the material are those of the authors, and not necessarily those of $\mathrm{ClHI}$. This study is also based in part on data provided by the Better Outcomes Registry and Network (BORN), part of the Children's Hospital of Eastern Ontario. The interpretation and conclusions contained herein do not necessarily represent those of BORN Ontario.

Accepted: Apr. 14, 2020

Correspondence to: Ziv Harel, Ziv.Harel@unityhealth.to 